\title{
Preoperative counseling and expectation management for inflatable penile prosthesis implantation
}

\author{
Gopal L. Narang ${ }^{1}$, Bradley D. Figler ${ }^{1}$, Robert M. Coward ${ }^{1,2}$ \\ ${ }^{1}$ Department of Urology, UNC School of Medicine, Chapel Hill, NC, USA; ${ }^{2}$ UNC Fertility, LLC, Raleigh, NC, USA \\ Contributions: (I) Conception and design: GL Narang, RM Coward; (II) Administrative support: GL Narang, RM Coward; (III) Provision of study \\ materials or patients: GL Narang, RM Coward; (IV) Collection and assembly of data: GL Narang, RM Coward; (V) Data analysis and interpretation: \\ All authors; (VI) Manuscript writing: All authors; (VII) Final approval of manuscript: All authors. \\ Correspondence to: Robert M. Coward, MD. 2113 Physicians Office Building, Campus Box 7235, Chapel Hill, NC 27599, USA. Email: MCoward@med.unc.edu.
}

\begin{abstract}
The inflatable penile prosthesis (IPP) is the gold standard surgical treatment for medical refractory erectile dysfunction (ED). While the modern IPP has enjoyed high satisfaction rates as a product of its continued innovation, reliability, and performance, patient dissatisfaction can still occur. IPP implantation restores physiologic function with cosmetic and psychological consequences, both of which place inherent emphasis on preoperative counseling and expectation management. This review aims to highlight the complex nature of such counseling and provide practitioners with a roadmap to navigate the landscape. Preoperative counseling begins with appropriate patient selection and identification of those patients who are at risk for dissatisfaction as a result of personality characteristics. The informed consent provides a natural framework to discuss the host of complications and risks that are associated with surgery, including infection, device malfunction, damage to nearby structures, and device erosion. Device selection is a nuanced process that merges patient preference with clinical factors and consideration. We address device selection through a description of cylinder construction, pump design, and reservoir placement in the context of preoperative counseling. Lastly, we draw attention to expectation management with a specific focus on possible post-operative changes to penile length and sensation as well as partner involvement. The modern IPP provides excellent results with high patient and partner satisfaction. Ultimately, satisfaction is dependent on multiple factors, but providing accurate, realistic counseling and expectation management prepares patients for the best possible outcomes.
\end{abstract}

Keywords: Expectation management; inflatable penile prosthesis (IPP); preoperative counseling

Submitted Mar 22, 2017. Accepted for publication Jun 27, 2017.

doi: 10.21037/tau.2017.07.04

View this article at: http://dx.doi.org/10.21037/tau.2017.07.04

\section{Introduction}

The inflatable penile prosthesis (IPP) is a vital piece in the framework of erectile dysfunction (ED) management. The modern prosthesis has been around for over 40 years and undergone numerous iterations. For patients refractory to medical therapies, including phosphodiesterase type 5 inhibitors, penile prosthesis placement is widely considered the gold standard surgical therapy $(1,2)$. The IPP has evolved from the semi-rigid implant and two-piece inflatable prosthesis to the modern three-piece inflatable device, the "Cadillac" of available penile implants. The modern IPP is a testament to surgical innovation, reliability, and patient satisfaction.

Though the IPP generally enjoys very high satisfaction rates, less-than-perfect outcomes and post-operative dissatisfaction can still occur. The primary goal of IPP placement is the restoration of physiologic function; however, it also carries cosmetic and psychosocial consequences. Given these considerations, there is always the risk of a disconnect between preoperative expectations and post-operative reality. These factors, in addition to the elective nature of the procedure, place a unique emphasis 
on preoperative counseling and expectation management.

Preoperative counseling is the primary method for limiting unrealistic expectations, optimizing post-operative satisfaction, and preparing patients for possible complications. The goal of this review is to explore preoperative counseling and expectation management for IPP placement. We will highlight areas to address within the preoperative assessment, discuss the decision of device selection, and focus on the dynamics of expectation management.

\section{Patient selection}

Selecting patients for IPP placement is typically straightforward, although there are many nuances that require significant consideration. Surgery has long been reserved for those with ED refractory to conservative therapy; however, this heterogeneous group includes patients with ED as a result of radical prostatectomy, diabetes and other obesity-related co-morbidities, priapism, neurological conditions, and Peyronie's disease (3). Such patients have unique individual considerations for counseling, expectations, and management. These patients have a variety of unique clinical and psychosocial factors that influence surgical risk, operative complexity, and postoperative satisfaction. Patients with body mass index $>30$, those who have undergone radical prostatectomy, and those who suffer from Peyronie's disease are at increased risk for post-operative dissatisfaction (4). Understanding which groups are at increased risk for more challenging outcomes allows for counseling and expectation management to start at the initial patient encounter.

Identifying the "difficult patient" is also critical. Difficult patients carry psychosocial variables that affect operative success and long term satisfaction. Trost et al. examined the cosmetic literature and identified seven characteristics associated with higher rates of postoperative dissatisfaction (5). These traits are represented in the mnemonic "CURSED Patient", which stands for Compulsive, Unrealistic, Revision, Surgeon Shopping, Entitled, Denial, and Psychiatric. Such characteristics may not be immediately apparent but with a focused discussion quickly rise to the top. Those who are compulsive or harbor unrealistic expectations are at risk for post-operative dissatisfaction due to a desire for the "perfect" surgery. Patients who seek surgical revision are not only at risk for dissatisfaction but also have higher rates of infections and decreased penile length (6). Within this subset of patients, approximately $16 \%$ seek revision for reasons outside of mechanical dysfunction or erosion, and such patients may be unsatisfied with their prior IPP experience and are primed for dissatisfaction (3). Entitled, or narcissistic, patients are particularly difficult because they require excessive time and resources, and they frequently have difficulty with post-operative compliance. The majority of patients that seek IPP placement do not display the abovementioned characteristics; however, identifying the patients who may is paramount in optimizing operative success and patient satisfaction.

\section{Preoperative counseling}

\section{Informed consent}

The informed consent provides a formal platform for preoperative counseling. A detailed explanation of the risks of surgery should be tailored to each patient based on their clinical picture. A short guide, provided by the Sexual Medicine Society of North America (SMSNA), has been developed to focus the informed consent discussion (7). The guide briefly touches on infection, device reliability, damage to nearby structures, device erosion as well as changes in penile length and sensation. This provides a useful framework for physicians to discuss preoperative counseling and expectations (Table 1).

\section{Infection}

The risk of infection with virgin implantation of the modern IPP has ranged from less than $1 \%$ and up to $4 \%$ $(1,9,10)$. Though the risk is low, patients should be aware of factors that predispose them to higher rates of infection and how to minimize their risk.

Patients with spinal cord injuries and long term steroid use are at increased risk of infection as well as those undergoing IPP revision (10-12). In the past, diabetes mellitus was believed to confer an elevated risk of infection, yet recent data challenges this assumption (13). Christodoulidou and colleagues performed a systematic review examining the rate of IPP infections in patients with diabetes mellitus. Their findings revealed no statistically significant increase in the risk of infection in patients with diabetes mellitus (14). Conflicting data also exists regarding the value of tight glucose control in decreasing the risk of infection in diabetic patients; however, patients should certainly be encouraged to keep their blood glucose values within normal range prior to surgery $(15,16)$. The AMS Inhibizone coating 
Table 1 Preoperative counseling and expectation management for IPP placement

\begin{tabular}{ll}
\hline Framework & Overview \\
\hline Patient selection (5) & Identify concerning patient factors and the "CURSED" patient \\
& Initial evaluation of expectations \\
Preoperative optimization & Evaluate for pertinent operative risk factors \\
& Discuss smoking cessation and glucose control \\
& For patients with length concerns, discuss preoperative vacuum or penile traction therapy \\
Informed consent (7) & Formal platform to discuss and counsel patient on operative risk, infections and device reliability \\
Device selection & Discussion of clinical factors that may effect device selection \\
Postoperative expectations & Highlight appropriate AMS/Coloplast devices \\
Partner involvement (8) & Candid discussion regarding postoperative changes to penile length and sensation \\
& Encourage partner participation and involvement from the initial visit \\
& Discuss implications of IPP placement on the dynamics of sexual intercourse \\
& Discuss potential benefits of sex therapy for interested couples \\
\hline
\end{tabular}

of rifampin and minocycline has been FDA approved to reduce implant revisions due to infection including diabetic patients. Mulcahy demonstrated an infection rate of $1.5 \%$ in over 6,000 antibiotic impregnated devices implanted in diabetic patients compared to $4.2 \%$ in non-coated devices, over a 7 -year period (17). Cessation from smoking prior to surgery can minimize the risk of infection. A meta-analysis by Sørensen et al. demonstrated that smokers undergoing surgery had an increased infection and wound complication rate with odds ratios of 1.79 and 2.07, respectively (18). Patients who quit smoking 4 weeks prior to surgery were able to reduce their risk of infection by an odds ratio of 0.49 .

Aside from optimizing patient factors, numerous advances in device construction, surgical technique, and antibiotic usage have decreased infection rates. One hallmark of the modern prosthesis is device coatings used to deter infection. AMS has developed the aforementioned InhibiZone ${ }^{\mathrm{TM}}$, an antibiotic impregnated coating with rifampin and minocycline, and Coloplast has a hydrophilic coating which absorbs antibiotics and inhibits bacterial adherence. Prior to their advent, the rate of prosthesis infection ranged from $3-10 \%$, and ever since the incidence has precipitously fallen $(16,19)$. In a retrospective analysis of over 36,000 post-market patient report forms submitted to Coloplast, Serefoglu et al. reported that at 11 years of follow-up, the rate of infection for antibiotic-coated devices was $1.4 \%$, compared to $4.6 \%$ for non-coated devices, $\mathrm{P}<0.001$ (20). Carson and colleagues reported similar data for antibiotic-impregnated devices, with a rate of device infection of $1.1 \%$ at a mean follow up of 7.7 years (21). The authors of this review prefer to quote patients in preoperative counseling that the rate of infection with virgin penile implants is "approximately $1 \%$ ".

Other advances in infection risk reduction include the use of the novel 'no-touch' technique pioneered by J. Francois Eid. Such techniques have not only been utilized in urologic surgery, but also orthopedic, plastic, breast, and neurosurgery. In regards to penile prosthesis placement, the 'no-touch' technique aims to limit the surgeon's contact with the patient's skin. The technique is accomplished by exchanging surgical instruments, frequently changing gloves, and innovative draping. Eid reported his results using the technique and demonstrated an impressive overall infection rate of $0.44 \%$ in a large retrospective cohort of over 2,500 antibiotic-impregnated implants over an 8-year period (22).

There is a paucity of data regarding the best regimen for antibiotic prophylaxis in the perioperative setting, and therefore a wide variety of algorithms and opinions exist. The current AUA Best Practice Policy Statement on Antimicrobial Prophylaxis recommends less than $24 \mathrm{~h}$ of antibiotic therapy after penile prosthesis insertion, citing data from the orthopedic literature (23). A panel of North American expert prosthetic surgeons recommends the use of post-operative antibiotics, of varying types, from 5-14 days after surgery (24). This panel recognizes the AUA recommendations but draws attention to the lack of data 
and reality that one infected prosthesis is one too many. Furthermore, recent data by Kavoussi et al. has illustrated that preoperative urine cultures correlate poorly-only $7 \%$ of the time-with the causative organism of infected prosthesis (25). This provides greater evidence that a broader approach to antibiotic prophylaxis may be needed. Regardless of the protocol, patients should be counseled on the small risk of post-operative prophylactic antibiotics as well as the current disagreement between available general clinical guidelines and expert opinion specific to penile implant surgery, versus the potential benefits and local surgeon preference and experience using post-operative antibiotic regimens.

\section{Device reliability}

The evolution of the modern IPP has made device malfunction much less common than in years past. Carson et al. examined the mechanical reliability of the AMS 700 CX and reported a 3 -year revision free rate of $92.1 \%$ (26). Wilson and colleagues examined data from 2,384 virgin implantations and estimated a 5-year mechanical survival of the AMS $700 \mathrm{CX}$ and Mentor Alpha 1, now Coloplast Titan, as $85.2 \%$ and $89.9 \%$, respectively $(27,28)$. The largest and most contemporary cohort of prospectively followed penile prosthesis cases found a re-operative rate of $3.9 \%$ at a median follow-up of approximately 8 years (29). Although mechanical failure is relatively infrequent, patients should understand that surgical correction and even device replacement may be required if it were to occur.

Patients undergoing IPP implantation for Peyronie's disease are at increased risk for malfunction or the need for revision. Chung et al. examined a cohort of patients with Peyronie's disease undergoing IPP implantation with modeling and found 5-year estimates of mechanical survival for the AMS 700 CX and Coloplast Titan of $91 \%$ and $87 \%$, respectively (30). Wilson et al. compared mechanical revision rates in patients with Peyronie's disease who performed simultaneous modeling with IPP with those receiving an IPP without a history of Peyronie's disease, and they reported a revision rate of $12.5 \%$ and $12.4 \%$, respectively, at 5 years (31). Furthermore, when examining the cohort of 104 Peyronie's patients and comparing implant type, of the 14 AMS 700 CX devices implanted, $28.6 \%$ suffered mechanical failure compared with $10 \%$ of the 90 Mentor Alpha I's implanted, and this difference was statistically significant $(\mathrm{P}=0.027)$.

Patients with Peyronie's disease should be counseled on modeling and its benefits toward penile straightening, but with an added modest increase in mechanical failure above what may be expected with virgin cases without the need for modeling (31).

\section{Damage to nearby structures}

The placement of the IPP requires purposeful disruption of the cavernous bodies; however, unintentional damage to the urethra, bladder, bowel and scrotal contents can rarely occur. Damage to the urethra is particularly worrisome, as it requires primary repair and abortion of the procedure. Rates of urethral perforation have been reported between $0.1-4.0 \%$ (32-35). Damage to the bladder is infrequent but can occur during traditional retropubic reservoir placement. Bowel perforation is also a rare complication; patients with prior pelvic surgery such as robotic prostatectomy, renal transplant, or laparoscopic inguinal hernia repair may be at greater risk due to prior disruption of the space of Retzius (36).

Damage to surrounding structures may also occur as a consequence of device erosion or migration. IPP erosion can involve the distal corpora, urethra, glans and other nearby structures. Broadly speaking, erosion has a reported incidence ranging from $1.0-6.0 \%(34,37)$. Minervini et al. reported their experience of over 504 IPP implantations and reported a corporal erosion rate of $5.4 \%$, a rate similar across malleable, two-piece inflatable, and three-piece IPP devices (34).

Patients with spinal cord injuries are known to have higher rates of IPP complications and erosion. Many spinal cord patients are dependent on clean intermittent catheterization for bladder management; frequent passage of the catheter per urethra may place them at high risk of urethral erosion. In a cohort of 48 patients with spinal cord injury who underwent malleable prosthesis insertion, two patients $(4.8 \%)$ suffered erosion, one each of urethral and glandular erosions, both requiring explantation of the device (37).

\section{Preoperative optimization}

Preoperative counseling not only includes a discussion of surgical risk but also medical optimization. As previously discussed, the importance of smoking cessation and appropriate glucose control should be reiterated. Patients with significant cardiac or pulmonary history should obtain appropriate preoperative evaluation and clearance.

In regards to penile length optimization, recent studies have endorsed the use of vacuum erection devices in the preoperative period to minimize post-operative losses $(38,39)$. Sellers et al. instituted a protocol of daily vacuum therapy 
for up to 2 months prior to surgery. They compared their protocol in over 750 patients to a cohort of 235 patients who received implants prior to protocol implementation. They demonstrated an increase in average implanted cylinder length of $3.63 \mathrm{~cm}(18.4$ to $22.03 \mathrm{~cm})$ between the two cohorts after institution of the vacuum therapy protocol (40). They posit that daily vacuum erection device usage and repetitive penile engorgement facilitates easier distal corporal dilation and allows for larger cylinder insertion.

Canguven and colleagues formally tested the efficacy of preoperative vacuum erection device use prior to IPP placement in a well-designed randomized control trial (41). They randomized 51 patients to a control arm and a treatment arm which consisted of 10-15 min per day of vacuum therapy for greater than 30 days. The treatment arm showed a statistically significant increase in day of surgery mean stretched penile length compared to the control arm, $0.80 \pm 0.38 \mathrm{~cm}(\mathrm{P}<0.05)$. Surgeons, blinded to both arms, subjectively reported smoother corporal dilation in the treatment group. More studies are needed to fully evaluate the long-term effects of preoperative vacuum therapy.

\section{Device explant}

All patients should be counseled on the possibility of device explanation. Reasons for device removal include infection, erosion, persistent discomfort or pain, and malfunction $(42,43)$. Explantation is a devastating result and, though never expected, the patient should be aware that it may be required if the circumstances arise. In regards to device removal for infection, the option for immediate salvage or replacement with a new device is a possibility. Mulcahy developed the salvage technique of sequential antibiotic washes and immediate replacement with a new device in 1996 (44). The benefit of such a technique is its success in removing the bacterial biofilm which may be a seed for infection in the future. In his initial series, 11 patients underwent salvage replacement and 91\% (10/11) were free from infection post-operatively, with a mean follow-up of 21 months (44). The benefits of washout are not only seen in an infected device. In a multicenter study examining 200 revision surgeries, Henry et al. demonstrated increased infectionfree survival after revision when a washout was performed for mechanical failure, $99 \%$ vs. $89 \%$ at 5 years (3). Revision and explant surgeries are also assisted by the development of antibiotic coated and impregnated coatings. Carson et al. demonstrated a $1.77 \%$ rate of revision due to infection in over 36,000 Inhibizone-coated prosthesis, compared to
$3.09 \%$ for uncoated prosthesis, over a 7-year period (45). The decision to perform a salvage or revision operation should be performed via shared decision making with the patient. Furthermore, replacement with a malleable prosthesis or multi-component device should also be discussed prior to surgery.

\section{Device selection}

\section{Device considerations}

Device selection is a cooperative decision between patient and surgeon that requires consideration and counseling. Clinical considerations such as the etiology of ED, penile length and anatomy, prior surgeries, manual dexterity, spinal cord injuries, and prior device explant all influence device recommendation (Table 2). Furthermore, surgeon preference and experience, device availability, and cost can also influence selection. Preoperative discussions should focus on familiarization with the prostheses and explanation of pertinent differences among available devices. Our discussion will center around pertinent differences between the two most commonly used three-piece prostheses, the AMS $700 \operatorname{series}^{\mathrm{TM}}$ (Figure 1) and Coloplast Titan ${ }^{\mathrm{TM}}$ (Figure 2).

\section{Cylinder construction}

The AMS 700 series $^{\mathrm{TM}}$ and Coloplast Titan ${ }^{\mathrm{TM}}$ differ in their cylinder construction. The AMS 700 series has a three-layer construction consisting of a woven Dacron-Lycra fabric in between two silicon layers. When woven unidirectionally, the Dacron-Lycra layer only enables girth expansion as seen in the CX and the narrower CXR cylinder models. The LGX model has a bidirectional weave, facilitating both length and girth expansion.

These differences in AMS cylinder design have clinical implications. For patients with Peyronie's disease, length expansion may exacerbate curvature, therefore making models restricted to girth expansion such as the CX and CXR more appropriate (28). Post-operative penile length is a significant satisfaction variable, and patients who choose the CX or CXR should understand the implications of the lack of length expansion (48).

The Coloplast Titan is constructed of a trademarked polymer, known as Bioflex, which is a polyurethane material that achieves girth expansion. Anecdotally, the Coloplast Titan cylinder has been perceived as more rigid and less compressible than the AMS 700. To test this observation, 
Table 2 Patient factors impact on device selection

\begin{tabular}{|c|c|c|}
\hline Patient factors & Preoperative considerations & Device selection \\
\hline $\begin{array}{l}\text { Limited manual } \\
\text { dexterity }(28,35)\end{array}$ & (I) Difficulty with device inflation/deflation & $\begin{array}{l}\text { (I) Malleable: (i) AMS Spectra; (ii) Coloplast Genesis } \\
\text { (II) Pump: (i) AMS Momentary Squeeze; (ii) Coloplast Titan }\end{array}$ \\
\hline $\begin{array}{l}\text { Prior pelvic surgery } \\
(35,46,47)\end{array}$ & $\begin{array}{l}\text { (I) Obliteration of the space of Retzius from robotic } \\
\text { prostatectomy, laparoscopic hernia, or renal transplant } \\
\text { (II) Consider ectopic reservoir placement }\end{array}$ & $\begin{array}{l}\text { (I) Two-piece IPP: AMS Ambicor } \\
\text { (II) Reservoirs: (i) AMS Conceal; (ii) Coloplast } \\
\text { Cloverleaf }\end{array}$ \\
\hline $\begin{array}{l}\text { Corporal fibrosis } \\
(1,35,46)\end{array}$ & $\begin{array}{l}\text { (I) Increased risk of infection, corporal perforation, and } \\
\text { urethral injury } \\
\text { (II) Difficulty with corporal dilation }\end{array}$ & $\begin{array}{l}\text { Narrow cylinder three-piece IPP: (i) AMS } 700 \text { CXR; } \\
\text { (ii) Coloplast Titan Narrow }\end{array}$ \\
\hline
\end{tabular}

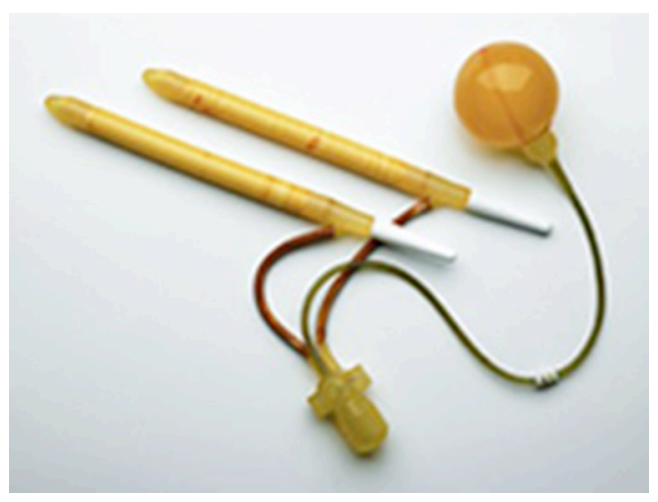

Figure 1 AMS $700^{\mathrm{TM}}$ LGX three-piece inflatable penile prosthesis with Inhibizone ${ }^{\mathrm{TM}}$.

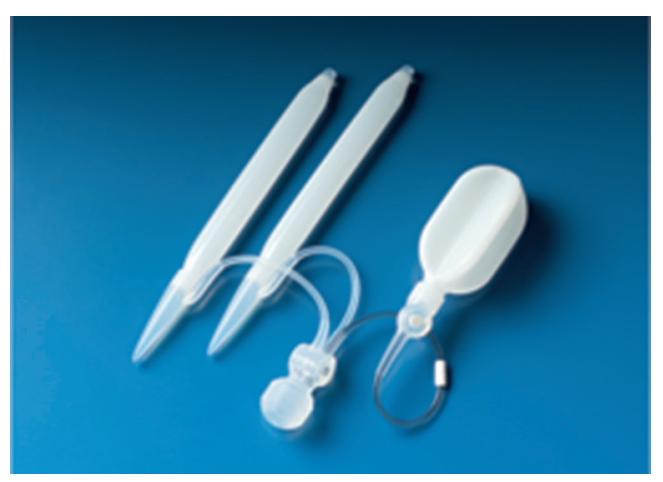

Figure 2 Coloplast Titan $^{\mathrm{TM}}$ Touch three-piece inflatable penile prosthesis.

(C) Translational Andrology and Urology. All rights reserved.
Scovell and colleagues evaluated longitudinal and horizontal load testing in ex vivo AMS 700 LGX and Coloplast Titan cylinders (49). Cantilever testing across various fill pressures demonstrated increased resistance to gravitational forces with the Coloplast Titan compared with the AMS 700 CX. This has important implications for patients with penile fibrosis who would benefit from a device more resistant to external forces. Furthermore, the increased resistance of the Coloplast Titan to horizontal loads, as compared with the AMS 700 LGX, makes it an ideal device for use in patients with Peyronie's disease to be used with concurrent penile modeling for straightening after implantation.

The AMS and Coloplast devices have distinct differences in their cylinder coatings. Both employ coatings to deter the development of infection. The AMS 700 implants have a patented Inhibizone ${ }^{\mathrm{TM}}$ coating which incorporates a proprietary combination of rifampin and minocycline into the lining of the device, coating the cylinder, tubing, pump and reservoir. This coating is responsible for the distinctive yellow color of the AMS 700 devices. The Inhibizone ${ }^{\mathrm{TM}}$ is FDA approved to reduce infections. The Inhibizone ${ }^{\mathrm{TM}}$ has been shown to reduce 60 -day infection rates by over $80 \%$ compared with uncoated devices (50). As previously mentioned Carson et al. demonstrated a $1.77 \%$ rate of revision due to infection in Inhibizone-coated prosthesis, compared to $3.09 \%$ for uncoated prosthesis (45). In regards to preoperative considerations, patients considering the AMS 700 series devices should be screened for allergies to 
tetracyclines or rifampin as these are contraindications to Inhibizone ${ }^{\mathrm{TM}}$ use.

The Coloplast Titan utilizes a polyvinylpyrrolidone (PVP) polymer, which is capable to absorbing antibiotics from a pre-determined dip and provides a hydrophilic coating that inhibits bacterial adherence. The PVP coating is novel in that the surgeon determines the antibiotic to use based on patient characteristics, allergies, and local hospital antibiogram. As previously stated, the hydrophilic coating has been shown to decrease infections compared with non-coated devices by $69 \%$, as illustrated by a $1.4 \%$ revision rate due to infection at 11 years follow up in coated devices compared with a $4.5 \%$ infection rate in noncoated implants (20). Both the AMS and Coloplast Titan offer cylinder coatings aimed at deterring infection and do so effectively. In a retrospective review of infection rates between Coloplast Titan devices, soaked in a rifampin and gentamicin coating, and Inhibizone impregnated AMS devices, infection rates were $4.4 \%$ and $1.3 \%$, respectively $(\mathrm{P}=0.05)$ (51). Patients should be counseled on the differences between both devices and screened for allergies that may preclude them from utilizing an implant with an impregnated antibiotic coating such as Inhibizone.

\section{Pump design}

Patients interact with the pump more than any other component of the IPP, making pump considerations integral to device selection. Recently, more sophisticated and smaller pumps have been designed which help facilitate device cycling.

The AMS 700 series $^{\mathrm{TM}}$ utilizes the momentary squeeze $(M S)^{\mathrm{TM}}$ pump. The MS pump features a one-touch release for simplified deflation and a slimmer profile compared to prior AMS pump models. It also includes a lock out value to protect against auto inflation. Knoll et al. evaluated the MS pump in a single arm, multicenter prospective study surveying both patients and physicians. They surveyed 69 patients who had the AMS 700 MS Pump implanted, and 94\% were able to deflate the device with a single activation of the button and $96 \%$ felt it was easily to locate (52). Furthermore, approximately $67 \%$ of the patients in the survey were able to be trained on the pump in less than $6 \mathrm{~min}$, and at 6 months, $77 \%$ were very satisfied with its use (52). In regards to physician opinion, over half of those surveyed by Knoll believed the MS pump was easier to implant compared to prior models.

The Coloplast Titan ${ }^{\mathrm{TM}}$ offers two pump designs: the one touch release (OTR) and the Titan Touch. The OTR pump was designed to allow for deflation to occur with a single push and has removed need for cylinder compression. The OTR pump has good mechanical reliability and has been shown to provide easy deflation. In a single arm, multicenter prospective study evaluating the Coloplast OTR pump in 113 patients, satisfaction was over $90 \%$, and easy deflation was endorsed by greater than $70 \%$ of patients at 6 and 12 months (53). Recently, Coloplast has released the Titan Touch pump, which also operates by a OTR mechanism. The Titan Touch has a smaller profile than the OTR pump due to minimization of the deflation pads. The ergonomics of the deflation pads have also changed, with a concave and dimpled surface added to allow for easier recognition of the button. The Titan Touch has quickly risen to prominence with the Coloplast product line, and given its preference among surgeons, it has become the standard pump option for Coloplast devices.

\section{Reservoir placement}

The reservoir has traditionally been placed into the retropubic space of Retzius. However, reservoir placement has evolved in recent years and currently may be influenced by patient factors. Prior pelvic surgery or radiation can complicate standard reservoir placement. In a survey to the members of the SMSNA, $82 \%$ of the 81 surgeons surveyed felt that patients with a prior history of radical prostatectomy sometimes or frequently made traditional reservoir placement more difficult (54). Furthermore, reservoir placement in the retropubic space carries the risk of bladder, vascular, or hollow viscus injury $(28,36,43)$. In the context of device selection, patients who are at elevated risk for injury during reservoir placement should understand that ectopic reservoir placement may be required. Both AMS and Coloplast have developed reservoirs with low profiles, the Conceal and Cloverleaf reservoirs, respectively, that facilitate ectopic placement (34). Possible ectopic sites include areas directed cephalad anterior or posterior to the transversalis fascia, and reservoir placement anterior to transversalis and posterior to the rectus abdominis muscle high within the abdominal wall was first described by Morey and is now referred to as "high sub-muscular" reservoir placement $(47,55)$. Ectopic placement carries the small risk of a palpable reservoir, although in a contemporary series of 146 patients who underwent a high sub-muscular reservoir placement, only $2(1.3 \%)$ were revised due to palpability (56). Coloplast received FDA approval for ectopic reservoir placement in 2015, which is still commonly called "ectopic," 
although now may be considered "on-label."

The current indications are extremely limited in most settings, but in the small subset of patients who cannot undergo retropubic or ectopic reservoir placement, one option still exists. The two-piece prosthesis is an alternative when there is a desire to avoid the reservoir altogether. An example would be a patient with a significant history of both pelvic and abdominal wall surgery, such as what might be encountered after low anterior rectal resection, bower diversion, and/or rectus flap reconstruction for colorectal cancer. The lack of a separate reservoir allows the two-piece prosthesis to circumvent difficulties seen with the reservoir placement in some at risk patients $(48,57)$. The only twopiece prosthesis on the market currently is the AMS Ambicor device. In older data, two-piece prostheses have low infection rate, good reliability and high patient-partner satisfaction $(1,46)$. Given the limited reservoir space built within the rear of the cylinders, the two-piece implant is not able to achieve the same rigidity as three-piece devices; however, it remains an option for select groups.

\section{Expectation management}

\section{Post-operative satisfaction}

Post-operative satisfaction is the product of numerous variables and is highly dependent on expectation management (58). Contemporary series have cited IPP satisfaction rates from 78-96\% (59-62). Satisfaction with IPP placement is a consequence of numerous variables. Carvalheira et al. analyzed patient satisfaction among a cohort of 47 men who underwent IPP placement and reported $79 \%$ satisfaction. When analyzing the reasons for satisfaction, they noted psychological factors, relational factors, improvements of sexual function, and improvement in voiding (63). Such research points to the multifaceted and complex nature of post-operative satisfaction.

Although the vast majority of patients are satisfied with IPP placement, minor dissatisfaction can occur. Poor surgical outcomes such as infection, erosion, device failure, or intraoperative complications clearly may affect satisfaction. Other than recognized complications, other factors exist which influence satisfaction. In the previously mentioned study from Carvalheira et al., patient dissatisfaction was attributed to decreased penile length, unrealistic expectations, unnatural feel, infrequent intercourse, delayed ejaculation, and partner dissatisfaction (63). Inappropriate assumptions regarding the penile prosthesis, its implantation, the resulting erection, or its effect on preexisting relationships may be causes for post-operative dissatisfaction (64).

It has been said by many high volume penile implant surgeons that when it comes to setting preoperative expectations for penile implant surgery, "under sell and over deliver." Approximately $75 \%$ of malpractice claims against surgeons are a product of dissatisfaction and misunderstandings after surgery, likely a consequence of poor communication (65). Preoperative counseling initiates the discussion and helps to frame expectations. As previously examined here in this review, highlighting the risks of device implantation and appropriate device selection are the initial steps. Focused communication regarding changes in penile length and sensation as well as the dynamics of partner satisfaction are the next key steps in expectation management.

\section{Penile length and sensation}

One of the most common reasons for post-operative dissatisfaction after penile prosthesis implantation is perceived loss of penile length (66). Over $70 \%$ of patients endorse a loss in length, even in the absence of measurable evidence (48). Preoperative stretched length provides a realistic expectation for post-operative results (67). Patients should be counseled on post-operative length and understand that IPP placement will help restore rigidity but not augment length, even when the lengthening cylinder of the AMS 700 LGX is chosen. Strategies to maintain length, such as preoperative vacuum erection device use, have been proposed and may benefit overly concerned patients. While the authors of this review do not routinely recommend it, a suggestion of a short period of preoperative vacuum therapy or penile traction for certain patients prior to penile implant surgery may facilitate active participation on the patient's part to help them achieve what they perceive to be the maximum possible length. Patients with a history of radical prostatectomy, corporal fibrosis from such conditions as priapism or intracavernosal injections, and Peyronie's disease are at increased risk of penile shortening and may require additional focused counseling (43).

Changes in penile sensation should also be addressed preoperatively. Poor glandular engorgement after prosthesis implantation can affect patient and partner satisfaction (66). The use of intra-urethral alprostadil may be an effective therapeutic option for some patients lacking glandular engorgement (68). Some may experience an unnatural feeling with intercourse which can also influence satisfaction (69). 
This can improve with time, and additionally may improve with appropriate sex or couple's therapy. Ultimately, setting appropriate preoperative expectations regarding penile length and sensation is the best way to limit post-operative dissatisfaction for these common complaints.

\section{Partner satisfaction}

Researchers have shown that satisfaction after surgery is influenced by both the patient and the partner. Gittens and colleagues evaluated patient and partner satisfaction after IPP placement using patient surveys and demonstrated $77.8 \%$ and $78.1 \%$ satisfaction, respectively (59). When further examining the relationship, they found that patients who were more satisfied with their implant had statistically significant higher partner satisfaction scores, compared with men reporting dissatisfaction with their device. Interestingly, patients who were dissatisfied with their implant were more likely to have partners with low female sexual functional scores (70). This observation highlights the importance of counseling the female partner prior to placement of the IPP in order to assess female partner sexual dysfunction and libido.

Such a study speaks to the multifaceted and interconnected nature of patient and partner satisfaction. Partners are often overlooked during preoperative counseling and unaware of changes that may occur with implantation. These changes, such as decreased penile length, girth, and glandular rigidity can affect a partner's sexual experience. Furthermore, IPP implantation alters the dynamics of intercourse, as prosthesis inflation and deflation need to be incorporated seamlessly and may prove difficult for some patients.

Involving partners early in preoperative counseling may help optimize the post-operative experience. Counseling may facilitate communication and help set appropriate expectation. Patients and partners may also benefit from pre-surgical sex therapy, focusing on increasing sexual communication and offering strategies to apply postoperatively (8).

\section{Conclusions}

Preoperative counseling is a dynamic process that begins at the first visit and continues until the patient enters the operating room. Counseling begins with optimization of co-morbid medical conditions and identifying patients who are at increased risk for more challenging outcomes, including the "difficult" patient who may require additional counseling prior to surgery. Informed consent prior to surgery must include detailed discussions about risks that are unique to penile implant surgery, including infection and device malfunction. A detailed discussion of the available device options including the nuanced differences is important for the patient and surgeon to find the most appropriate fit for each patient. Lastly, expectation management with a detailed discussion of penile length, sensation, and patient and partner satisfaction is paramount to having a satisfied patient after placement of an IPP. Providing accurate, realistic expectations ultimately prepares patients for the best possible outcomes.

\section{Acknowledgements}

None.

\section{Footnote}

Conflicts of Interest: RM Coward has received compensation for consulting services from both Coloplast Inc. and Boston Scientific. The other authors have no conflicts of interest to declare.

\section{References}

1. Trost L, Wanzek P, Bailey G. A practical overview of considerations for penile prosthesis placement. Nat Rev Urol 2016;13:33-46.

2. Pisano F, Falcone M, Abbona A, et al. The importance of psychosexual counselling in the re-establishment of organic and erotic functions after penile prosthesis implantation. Int J Impot Res 2015;27:197-200.

3. Henry GD, Donatucci CF, Conners W, et al. An outcomes analysis of over 200 revision surgeries for penile prosthesis implantation: A multicenter study. J Sex Med 2012;9:309-15.

4. Akin-Olugbade O, Parker M, Guhring P, et al. Determinants of patient satisfaction following penile prosthesis surgery. J Sex Med 2006;3:743-8.

5. Trost LW, Baum N, Hellstrom WJ. Managing the Difficult Penile Prosthesis Patient. J Sex Med 2013;10:893-906.

6. Caire AA, Boonjindasup A, Hellstrom WJ. Does a replacement or revision of an inflatable penile prosthesis lead to decreased patient satisfaction? Int J Impot Res 2011;23:39-42.

7. Penile prosthesis information form, 2008. Available online: http://www.smsna.org/V1/images/SMSNAIPP-policy-ws.pdf 
8. Schover LR, von Eschenbach AC. Sex therapy and the penile prosthesis: a synthesis. J Sex Marital Ther 1985;11:57-66.

9. Mandava SH, Serefoglu EC, Freier MT, et al. Infection retardant coated inflatable penile prostheses decrease the incidence of infection: A systematic review and meta-analysis. J Urol 2012;188:1855-60.

10. Wilson SK, Zumbe J, Henry GD, et al. Infection reduction using antibiotic-coated inflatable penile prosthesis. Urology 2007;70:337-40.

11. Kava BR, Kanagarajah P, Ayyathurai R. Contemporary revision penile prosthesis surgery is not associated with a high risk of implant colonization or infection: A singlesurgeon Series. J Sex Med 2011;8:1540-6.

12. Collins KP, Hackler RH. Complications of penile prostheses in the spinal cord injury population. J Urol 1988;140:984-5.

13. Wilson SK, Delk JR 2nd. Inflatable Penile Implant Infection: Predisposing Factors and Treatment Suggestions. J Urol 1995;153:659-61.

14. Christodoulidou M, Pearce I. Infection of Penile Prostheses in Patients with Diabetes Mellitus. Surg Infect (Larchmt) 2016;17:2-8.

15. Bishop JR, Moul JW, Sihelnik SA, et al. Use of glycosylated hemoglobin to identify diabetics at high risk for penile periprosthetic infections. J Urol 1992;147:386-8.

16. Wilson SK, Carson CC, Cleves MA, et al. Quantifying risk of penile prosthesis infection with elevated glycosylated hemoglobin. J Urol 1998;159:1537-9; discussion 1539-40.

17. Mulcahy JJ, Carson CC. Long-term infection rates in diabetic patients implanted with antibiotic-impregnated versus nonimpregnated inflatable penile prostheses: 7-year outcomes. Eur Urol 2011;60:167-72.

18. Sørensen LT. Wound healing and infection in surgery. The clinical impact of smoking and smoking cessation: a systematic review and meta-analysis. Arch Surg 2012;147:373-83.

19. Pineda M, Burnett AL. Penile Prosthesis Infections-A Review of Risk Factors, Prevention, and Treatment. Sex Med Rev 2016;4:389-98.

20. Serefoglu EC, Mandava SH, Gokce A, et al. Long-Term Revision Rate due to Infection in Hydrophilic-Coated Inflatable Penile Prostheses: 11-Year Follow-up. J Sex Med 2012;9:2182-6.

21. Carson CC, Mulcahy JJ, Harsch MR. Long-term infection outcomes after original antibiotic impregnated inflatable penile prosthesis implants: $\mathrm{Up}$ to 7.7 years of followup. $\mathrm{J}$ Urol 2011;185:614-8.

22. Eid JF. Penile Implant: Review of a "No-Touch"
Technique. Sex Med Rev 2016;4:294-300.

23. Wolf JS, Bennett CJ, Dmochowski RR, et al. Best practice policy statement on urologic surgery antimicrobial prophylaxis. J Urol 2008;179:1379-90.

24. Darouiche RO, Bella AJ, Boone TB, et al. North American consensus document on infection of penile prostheses. Urology 2013;82:937-42.

25. Kavoussi NL, Siegel JA, Viers BR, et al. Preoperative Urine Culture Results Correlate Poorly With Bacteriology of Urologic Prosthetic Device Infections. J Sex Med 2017;14:163-8.

26. Carson CC, Mulcahy JJ, Govier FE. Efficacy, Safety and Patient Satisfaction Outcomes of hte AMS 700CX Inflatable Penile Prosthesis: Results of a Long-Term Multicenter Study. J Urol 2000;164:376-80.

27. Wilson SK, Delk JR, Salem EA, et al. Long-term survival of inflatable penile prostheses: single surgical group experience with 2,384 first-time implants spanning two decades. J Sex Med 2007;4:1074-9.

28. Garber BB. Inflatable penile prostheses for the treatment of erectile dysfunction: an update. Expert Rev Med Devices 2008;5:133-44.

29. Onyeji IC, Sui W, Pagano MJ, et al. Impact of Surgeon Case Volume on Reoperation Rates after Inflatable Penile Prosthesis Surgery. J Urol 2017;197:223-9.

30. Chung E, Solomon M, Deyoung L, et al. Comparison between AMS 700 CX and Coloplast Titan Inflatable Penile Prosthesis for Peyronie's Disease Treatment and Remodeling: Clinical Outcomes and Patient Satisfaction. J Sex Med 2013;10:2855-60.

31. Wilson SK, Cleves MA, Delk JR 2nd. Long-term followup of treatment for Peyronie's disease: modeling the penis over an inflatable penile prosthesis. J Urol 2001;165:825-9.

32. Chung E, Van CT, Wilson I, et al. Penile prosthesis implantation for the treatment for male erectile dysfunction: Clinical outcomes and lessons learnt after 955 procedures. World J Urol 2013;31:591-5.

33. Anele UA, Le BV, Burnett AL. Suprapubic cystostomy for the management of urethral injuries during penile prosthesis implantation. Sex Med 2014;2:178-81.

34. Minervini A, Ralph DJ, Pryor JP. Outcome of penile prosthesis implantation for treating erectile dysfunction: Experience with 504 procedures. BJU Int 2006;97:129-33.

35. Levine LA, Becher E, Bella A, et al. Penile Prosthesis Surgery: Current Recommendations From the International Consultation on Sexual Medicine. J Sex Med 2016;13:489-518.

36. Vakalopoulos I, Kampantais S, Gkagkalidis K, et al. 
Complications of Inflatable Penile Prostheses Implantation Classified according to the Modified Clavien System. Adv Urol 2014;2014:5.

37. Kim YD, Yang SO, Lee JK, et al. Usefulness of a malleable penile prosthesis in patients with a spinal cord injury. Int J Urol 2008;15:919-23.

38. Pahlajani G, Raina R, Jones S, et al. Vacuum Erection Devices Revisited: Its Emerging Role in the Treatment of Erectile Dysfunction and Early Penile Rehabilitation Following Prostate Cancer Therapy. J Sex Med 2012;9:1182-9.

39. Wang R, Lin H, Wang G. Vacuum erectile device for penile rehabilitation. J Integr Nephrol Androl 2014;1:4-10.

40. Sellers T, Dineen M, Salem EA, et al. Vacuum Preparation, Optimization of Cylinder Length and Postoperative Daily Inflation Reduces Complaints of Shortened Penile Length Following Implantation of Inflatable Penile Prosthesis. Adv Sex Med 2013;189:14-8.

41. Canguven O, Talib RA, Campbell J, et al. Is the daily use of vacuum erection device for a month before penile prosthesis implantation beneficial? a randomized controlled trial. Andrology 2017;5:103-6.

42. Montague DK. Pain and Inflammation Associated with Genitourinary Prostheses. In: Potts JM. edtor. Genitourinary Pain And Inflammation. Current Clinical Urology. Humana Press, 2008:385-91.

43. Mulcahy JJ, Kramer A, Brant WO, et al. Current management of penile implant infections, device reliability, and optimizing cosmetic outcome. Curr Urol Rep 2014;15:413.

44. Brant MD, Ludlow JK, Mulcahy JJ. The prosthesis salvage operation: immediate replacement of the infected penile prosthesis. J Urol 1996;155:155-7.

45. Carson CC, Mulcahy JJ, Harsch MR. 7-year infection related revision rates for naive inflatable penile prosthesis implants: Antibiotic impregnated vs. non-impregnated. J Urol 2010;183:e488.

46. Levine LA, Estrada CR, Morgentaler A. Mechanical reliability and safety of, and patient satisfaction with the Ambicor inflatable penile prosthesis: results of a 2 center study. J Urol 2001;166:932-7.

47. Perito P, Wilson S. The History of Nontraditional or Ectopic Placement of Reservoirs in Prosthetic Urology. Sex Med Rev 2016;4:190-3.

48. Deveci S, Martin D, Parker M, et al. Penile Length Alterations Following Penile Prosthesis Surgery. Eur Urol 2007;51:1128-31.

49. Scovell JM, Ge L, Barrera EV, et al. Longitudinal and Horizontal Load Testing of Inflatable Penile Implant Cylinders of Two Manufacturers: An Ex Vivo Demonstration of Inflated Rigidity. J Sex Med 2016;13:1750-7.

50. Carson CC. Efficacy of antibiotic impregnation of inflatable penile prostheses in decreasing infection in original implants. J Urol 2004;171:1611-4.

51. Dhabuwala C, Sheth S, Zamzow B. Infection Rates of Rifampin/Gentamicin-Coated Titan Coloplast Penile Implants. Comparison with Inhibizone-Impregnated AMS Penile Implants. J Sex Med 2011;8:315-20.

52. Knoll LD, Henry G, Culkin D, et al. Physician and patient satisfaction with the new AMS 700 momentary squeeze inflatable penile prosthesis. J Sex Med 2009;6:1773-8.

53. Ohl DA, Brock G, Ralph D, et al. Prospective Evaluation of Patient Satisfaction, and Surgeon and Patient Trainer Assessment of the Coloplast Titan One Touch Release Three-Piece Inflatable Penile Prosthesis. J Sex Med 2012;9:2467-74.

54. Karpman E, Sadeghi-Nejad H, Henry G, et al. Current opinions on alternative reservoir placement for inflatable penile prosthesis among members of the sexual medicine society of North America. J Sex Med 2013;10:2115-20.

55. Morey AF, Cefalu CA, Hudak SJ. High Submuscular Placement of Urologic Prosthetic Balloons and Reservoirs via Transscrotal Approach. J Sex Med 2013;10:603-10.

56. Chung PH, Morey AF, Tausch TJ, et al. High submuscular placement of urologic prosthetic balloons and reservoirs: 2-year experience and patient-reported outcomes. Urology 2014;84:1535-40.

57. Sadeghi-Nejad H, Fam M. Penile prosthesis surgery in the management of erectile dysfunction. Arab J Urol 2013;11:245-53.

58. Kramer AC, Schweber A. Patient expectations prior to coloplast titan penile prosthesis implant predicts postoperative satisfaction. J Sex Med 2010;7:2261-6.

59. Gittens P, Moskovic DJ, Avila D Jr, et al. Favorable female sexual function is associated with patient satisfaction after inflatable penile prosthesis implantation. J Sex Med 2011;8:1996-2001.

60. Bettocchi C, Palumbo F, Spilotros M, et al. Patient and partner satisfaction after AMS inflatable penile prosthesis implant. J Sex Med 2010;7:304-9.

61. Garber BB. Mentor alpha 1 inflatable penile prosthesis: Patient satisfaction and device reliability. Urology 1994;43:214-7.

62. Trost L, Hellstrom WJ. History, contemporary outcomes, and future of penile prostheses: A review of the literature. 
Sex Med Rev 2013;1:150-63.

63. Carvalheira A, Santana R, Pereira NM. Why Are Men Satisfied or Dissatisfied with Penile Implants? A Mixed Method Study on Satisfaction with Penile Prosthesis Implantation. J Sex Med 2015;12:2474-80.

64. Ulloa EW, Silberbogen AK, Brown K. Preoperative Psychosocial Evaluation of Penile Prosthesis Candidates Erin. Am J Mens Health 2008;2:68-75.

65. Tasman AJ. The psychological aspects of rhinoplasty. Curr Opin Otolaryngol Head Neck Surg 2010;18:290-4.

66. Montorsi F, Rigatti P, Carmignani G, et al. AMS threepiece inflatable implants for erectile dysfunction: a longterm multi-institutional study in 200 consecutive patients. Eur Urol 2000;37:50-5.

Cite this article as: Narang GL, Figler BD, Coward RM. Preoperative counseling and expectation management for inflatable penile prosthesis implantation. Transl Androl Urol 2017;6(Suppl 5):S869-S880. doi: 10.21037/tau.2017.07.04
67. Osterberg EC, Maganty A, Ramasamy R, et al. Pharmacologically induced erect penile length and stretched penile length are both good predictors of post-inflatable prosthesis penile length. Int J Impot Res 2014;26:128-31.

68. Mulhall JP, Jahoda A, Aviv N, et al. The impact of sildenafil citrate on sexual satisfaction profiles in men with a penile prosthesis in situ. BJU Int. 2004;93:97-9.

69. Porena M, Mearini L, Mearini E, et al. Penile prosthesis implantation and couple's satisfaction. Urol Int 1999;63:185-7.

70. Wiegel M, Meston C, Rosen R. The Female Sexual Function Index (FSFI): Cross-Validation and Development of Clinical Cutoff Scores. J Sex Marital Ther 2005;31:1-20. 